\title{
Fast NGC: A new on-line technique for fuel flow measurement
}

\author{
Felix Leach ${ }^{1}$, Martin Davy ${ }^{1}$, Manus Henry ${ }^{1}$, Maruthi Rochishnu Malladi ${ }^{1}$, Michael Tombs ${ }^{1}$, Feibiao \\ Zhou $^{1}$, Martin Gold ${ }^{2}$, Richard Pearson ${ }^{2}$
}

1. Department of Engineering Science, University of Oxford, UK

2. BP International Ltd. UK

SAE Technical Paper - Author's Accepted Manuscript

\begin{abstract}
Knowledge of fuel mass injected in an individual cycle is important for engine performance and modelling. Currently direct measurements of fuel flow to individual cylinders of an engine are not possible on-engine or in real-time due to a lack of available appropriate measurement techniques. The objective of this work was to undertake real-time Coriolis fuel flow measurement using GDI injectors on a rig observing fuel mass flow rate within individual fuel injections. This paper evaluates the potential of this technology - combining Coriolis Flow Meters (CFMs) with Prism signal processing together known as Fast Next Generation Coriolis (Fast NGC), and serves as a basis for future transitions on-engine applications. A rig-based feasibility study has been undertaken injecting gasoline through a GDI injector at 150 bar in both single shot mode and at a simulated engine speeds of 1788 and $2978 \mathrm{rpm}$. The results show that these injections can, in principle, be observed. In addition a number of features of the Fast NGC system unique to gasoline are discussed, and the repeatability of the technique is preliminarily assessed. The study concludes that the Fast NGC system has the potential to measure individual injector flow rates on-engine in real-time.
\end{abstract}

\section{Introduction}

Gasoline Direct Injection (GDI) engines are becoming the dominant technology for gasoline vehicles due to their lower $\mathrm{CO}_{2}$ emissions relative to Port Fuel Injected (PFI) engines [1]. GDI engines however are known to emit greater levels of particulate matter (PM) [2] as a result of inhomogeneities in the fuel-air mixing. These inhomogeneities are often the result of poor spray breakup on injection, which can lead to wall wetting and hence pool fires which lead to high levels of emitted PM [3]. At present GDI engine fuelling is controlled by knowledge of the air : fuel ratio $(\lambda)$, which is measured by sensors in the engine exhaust, and typically averaged over some or all of the cylinders of the engine (ranging from 3-12 cylinders for current production GDI engines). Such an approach can often mask cylinder-cylinder variations in fuelling, which can lead to cylinders which are overly rich in fuel, which will lead to high PM emissions.

Currently it is not possible to measure fuel mass flow rate on-engine in real-time. Typically injectors are characterised offline at given fuel pressures and temperatures, most commonly using the Bosch method [4] where the gasoline is injected into a filled tube, and the propagation of the pressure waves in that tube is analysed to obtain the mass flow rate [5]. Such measurements are also essential for modelling of GDI injectors and for computational fluid dynamics (CFD) validation [6].

Recently Volkswagen (VW) incorporated a new on-line method for determining injection rate into their new GDI engine family [7]. Here they use high speed fuel pressure measurements to infer mass flow rate of fuel and correct on a cylinder-cylinder basis for any differences in fuel flow rate. This approach requires a significant amount of calibration, due to fuel mass flow rate being a function of temperature, injector-injector interactions (which will in turn be dependent on engine speed and load), and in-cylinder conditions.

Fuel mass flow is measured using Coriolis meters in a large number of applications across a wide range of industry sectors from biomedical to upstream oil and gas [8]. In automotive applications Coriolis meters are typically used in research and development (R\&D) labs to measure total engine fuel flow at low pressure (averaged across all the cylinders of an engine) and are usually mounted some distance upstream of the engine, insulated from external vibrations. Coriolis Flow Meters (CFMs) consist of two distinct parts, the flow tube and the transmitter. The flow tube (often in a $U$ or omega shape) is oscillated by the transmitter at the natural frequency of a selected mode of vibration; a fluid flowing in the tube will undergo Coriolis acceleration which will lead to a phase difference from the forced original oscillation of the flowtube. By Newtonian analysis the time period corresponding to this phase difference is directly proportional to the mass flow rate. The transmitter initiates and maintains the oscillation of the flow tube, continuously monitoring via two (typically velocity) sensors, which are further used to determine phase difference. A more detailed discussion on the mechanics is included in [9]. While a variety of signal processing techniques have been used to track the essentially sinusoidal sensor signals in conventional Coriolis meters, a new technique, well suited to high noise rejection and fast measurement updates, is used here, based on Prism signal processing [10]. The process used to determine the phase from each sensor is outlined in Figure 1. The sensor outputs are pre-filtered with prism filters before a recursive signal tracker (RST) is used to calculate the phase $(\phi)$, amplitude $(A)$ and frequency $(f)$ of the each of the sensor outputs. Finally, the phase difference is calculated as $\phi_{1}-\phi_{2}$. Further details on the RST and the phase calculation are given in [10] 


\begin{tabular}{|c|c|c|c|c|}
\hline Sensor 1 & Pre-filtering & $\rightarrow$ & $\begin{array}{l}\text { Recursive } \\
\text { Signal }\end{array}$ & Sensor $1\left(\phi_{1}, A_{1}, f_{1}\right)$ \\
\hline Sensor 2 & filters & $\rightarrow$ & $\begin{array}{l}\text { Tracker } \\
\text { (RST) }\end{array}$ & Sensor $2\left(\phi_{2}, A_{2}, f_{2}\right)$ \\
\hline
\end{tabular}

Figure 1: Raw signal to phase calculation

Prism signal processing is a fully recursive Finite Impulse Response (FIR) filtering methodology which can be used for a variety of signal processing tasks. In particular, in this application, it is used to track frequencies of sinusoidal signals, and filter out undesired frequency components.

As discussed in [10], the combination of rapid fuel pulses and a mechanically noisy environment requires the removal of unwanted frequency components (including external vibration and undesired flow tube resonant modes) in order to obtain "clean" phase difference signals. More information about Prism and its applications can be found in [11-14].

So far the Prism signal processing coupled with Coriolis meters (together known as Fast Next Generation Coriolis - Fast NGC) has been successfully deployed on a diesel injection test rig tracking individual injections at pressures of up to 1000 bar and simulated engine speeds up to $4000 \mathrm{rpm}[9,10,12]$. These results showed a previously unseen phenomenon in which the measured phase difference (and hence mass flow rate) exhibited a dependence on the instantaneous flow tube phase, where the injection duration was shorter than the flow tube oscillation period. The aim of this work is to use, for the first time, the Fast NGC system on a GDI injector at conditions which are representative of those experienced in GDI engines.

\section{Experimental methodology}

In many ways the GDI application should be easier than the previous diesel testing. The injection pressures are substantially lower (350 bar is the upper limit for modern GDI systems [3], with $150 \mathrm{bar}$ more common), injection durations are typically longer, and there is no return line (or injector spill) on GDI injectors, unlike most of their diesel counterparts. However, the higher engine speeds in GDI engines, make the GDI application more challenging compared to diesel. In addition, the lack of pressure relief in the injector (injector spill) means that the hydraulic shocks at end of injection introduce higher levels of vibration into the fuel line, and hence a noisier environment for the Coriolis meter.

\section{Test rig}

A static GDI injector test rig has been used for all of the results reported in this work; the rig has been previously described in $[9,15]$. It uses a Bosch HDEV-5-1LE type multi-hole injector, of the same type fitted in production engines [16]. This injector can be run at fuel pressures up to $150 \mathrm{bar}$. The fuel was pressurised using an in-house air driven pressure accumulator and the injector was controlled and driven by a Bosch injector driver, enabled through National Instruments LabVIEW control software. A schematic of the rig is shown in Figure 2.

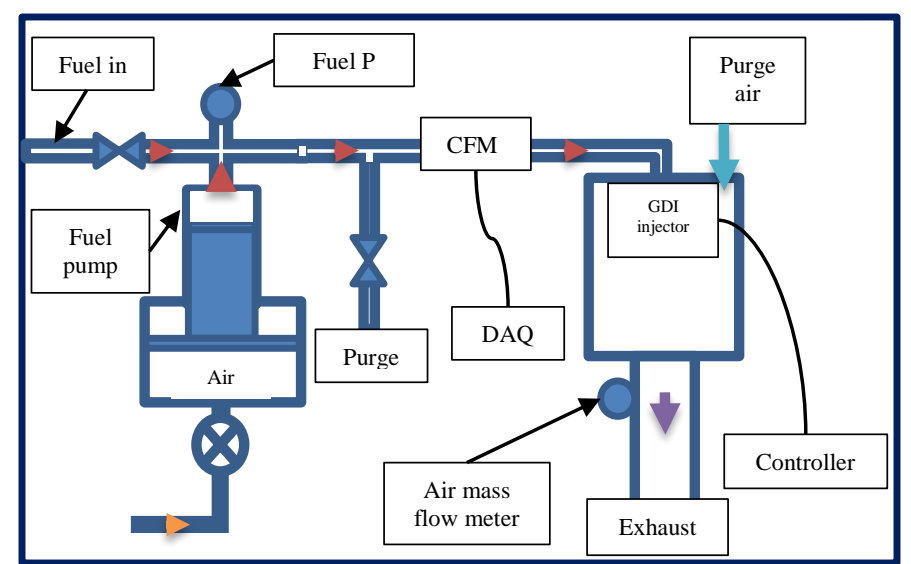

Figure 2: System schematic showing the injector rig with the Coriolis Flow Meter (CFM)

The Coriolis flow tube used was a RHM015 flow tube from Rheonik $\mathrm{GmbH}$, which has a natural drive mode frequency of $148.91 \mathrm{~Hz}$ when filled with fuel and operating at $150 \mathrm{bar}$ and at room temperature. The flow tube was controlled by a prototype transmitter, which was also used for data logging. The transmitter logs the flow tube data at $48 \mathrm{kHz}$, and applies Prism signal processing in real-time to give instantaneous mass flow rate measurement - no post-processing is needed. Initially the Prism filtering design is determined through characterising the system (any injection system will have its own natural modes of vibration etc.); subsequently, no further tuning is required.

\section{Test conditions}

Test conditions were selected to mimic typical GDI engine operation (within a homologation drive cycle envelope), as well as to test the operating envelope of the system. Previous work had uncovered the influence of flow tube phase on the measured mass flow [9] when the period of flow (i.e. fuel injection duration) is significantly shorter than the oscillation period of the flowtube; a much higher natural drive frequency (of the order of $\mathrm{kHz}$ ) will be required to overcome this issue. Currently there are no commercially available high frequency flow tubes rated at high enough pressures for this work. The design and manufacture of suitable bespoke flowtubes are planned, but for much of this work simulated engine speeds (for a four-stroke engine) were set to be multiples of the flowtube natural drive mode frequency $(149 \mathrm{~Hz})$ thus ensuring that injections were always taking place at the same point in the flow tube phase. The test conditions used in this work are shown in Table 1

Table 1: Experimental test conditions

\begin{tabular}{|l|l|l|l|}
\hline Simulated engine speed (rpm) & Single shot & 1788 & 2978 \\
\hline Injection frequency (Hz) & $\mathrm{n} / \mathrm{a}$ & 14.9 & 24.8 \\
\hline Injector ET (ms)* & $1 \& 2$ & 2 & $1,1.5, \& 2$ \\
\hline Injection pressure (bar) & 150 & 150 & 150 \\
\hline * Energising Time & & & \\
\hline
\end{tabular}


Total injected mass for each ET was measured by injecting $6 \times 300$ injections onto a mass balance and taking a mean. These results are shown in Table 2 .

Table 2: Total injected mass at each test condition

\begin{tabular}{|l|l|l|l|}
\hline Test condition & $1 \mathrm{~ms}$ ET & $1.5 \mathrm{~ms}$ ET & $2 \mathrm{~ms}$ ET \\
\hline Total injected mass $(\mathrm{mg} \pm \sigma)$ & $13.84 \pm 0.17$ & $20.89 \pm 0.12$ & $27.36 \pm 0.14$ \\
\hline
\end{tabular}

\section{Results}

Given our continuing investigation into the complex relationship between observed phase difference and true mass flow where the fuel pulse duration is significantly shorter than the oscillation period of the flowtube, experimental results presented here will be shown in terms of phase difference: the longer term goal (whether by improved modelling and/or faster flowtubes) is direct and accurate measurement of the instantaneous mass flow and total mass for each fuel injection event. For clarity, in these results, the injection durations will be presented as injector energising time (ET) only.

\section{Effect of external vibrations on flow meter}

A feature observed in undertaking this work was the additional mechanical vibration caused by not having an injector spill line on the GDI injector (unlike the previously tested diesel injectors). This introduced a substantial hydraulic shock into the high-pressure fuel line as the injector closed at end-of-injection. Such an increase in vibration made acquisition of the Coriolis flow data more challenging in this application than the authors had previously experienced and required careful mounting of the flow tube in order to minimise the effect of these vibrations on the flow tube. The flow tube was suspended with four rubber lined pipe clamps from a support structure which was stiffened with cross bracing. They were positioned such that there were two per side and are perpendicular to each other (vertical and horizontal orientation) in order to provide a stiff, vibration-isolated mounting for the flow tube.

As a result, a significant amount of extra Prism signal processing was needed. The upper part of Figure 3 shows the frequency spectrum of the raw sensor data. The flowtube natural drive mode frequency $(149 \mathrm{~Hz})$ is still highest peak in the spectrum. The so-called 'Coriolis' modes around $250 \mathrm{~Hz}$ are also significant. There is also high level noise occurring between $600 \mathrm{~Hz}$ and $800 \mathrm{~Hz}$. These additional vibration frequencies arise from yet other modes of the flowtube as well as from the experimental rig itself. The lower part of Figure 3 shows the frequency spectrum of the sensor data after the Prism filters have been applied. The Coriolis modes have been effectively suppressed while the region between $600-800 \mathrm{~Hz}$ has been reduced to a level similar to the noise floor at lower frequencies. Further filtering in this region could be applied but with limited additional benefit. The key to the design here is to selectively suppress the most significant error sources while preserving the dynamic response.
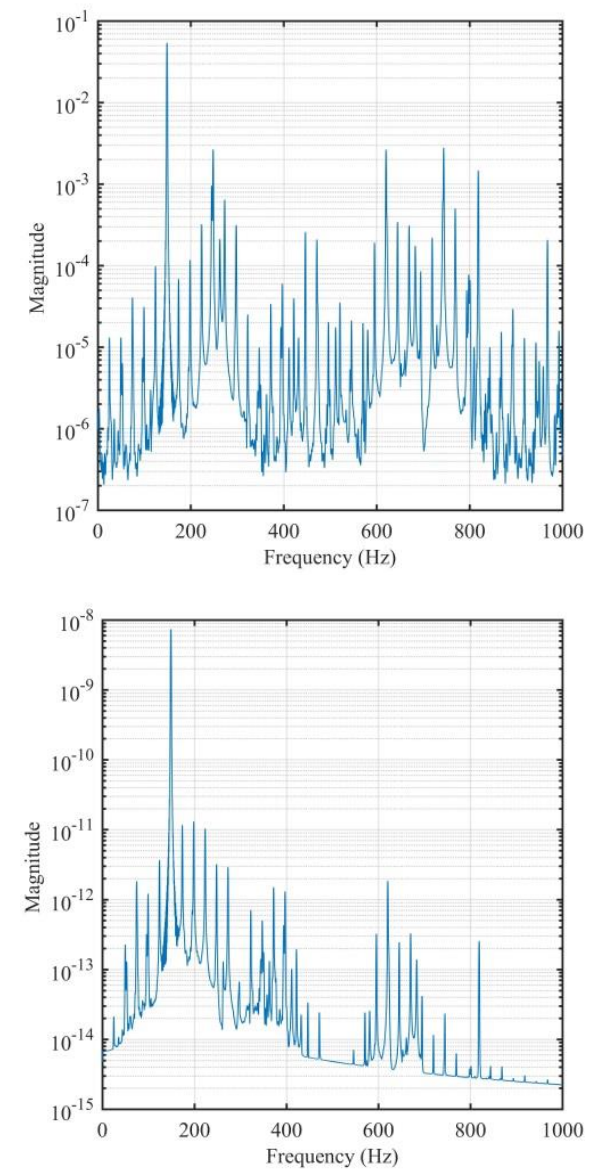

Figure 3: Unfiltered (upper) and filtered (lower) frequency spectra of the raw sensor data from the coriolis flowtube for $2 \mathrm{~ms}$ ET injections at $2978 \mathrm{rpm}$ equivalent engine speed

Figure 4 shows the effect of the Prism filtering on the measured phase difference (which is directly proportional to mass flow rate). Here it is seen that without using Prism notch filtering of undesired frequency components (grey line), no injections would be visible at all, whereas the effect of the Prism filtering is to show a clear train of $2 \mathrm{~ms}$ ET injection pulses at $2978 \mathrm{rpm}$ (blue line). However, compared to similar plots in the previously undertaken diesel tests (e.g. Figure 6 in [9]) it can be seen that the magnitude of the signal filtering required (i.e. overall amplitude reduction required to obtain a "clean" signal) is significantly higher, with much more noise observed here in Figure 4. This is attributed to the additional mechanical vibrations induced by hydraulic shock both at SOI and EOI. The detail behind the additional signal processing required will be discussed in a separate publication. 


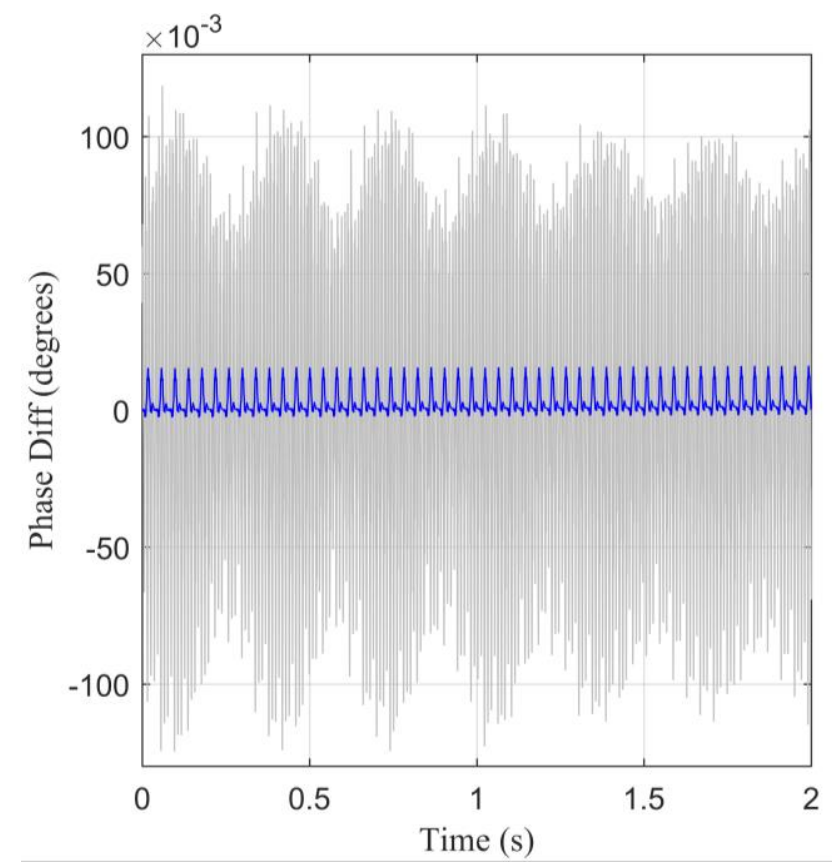

Figure 4: Comparison between raw and filtered CFM phase difference for $2 \mathrm{~ms}$ ET injections at $2978 \mathrm{rpm}$ equivalent engine speed

\section{Single shots}

Figure 5 shows the measured phase difference (which is directly proportional to mass flow rate) for a $1 \mathrm{~ms}$ ET single injection. For this short injection duration (and correspondingly small injected mass - $13.8 \mathrm{mg}$ ) the injection is clearly observed by the CFM via Prism signal processing. The performance of the meter and signal processing broadly matches those of the previous diesel injection experiments.

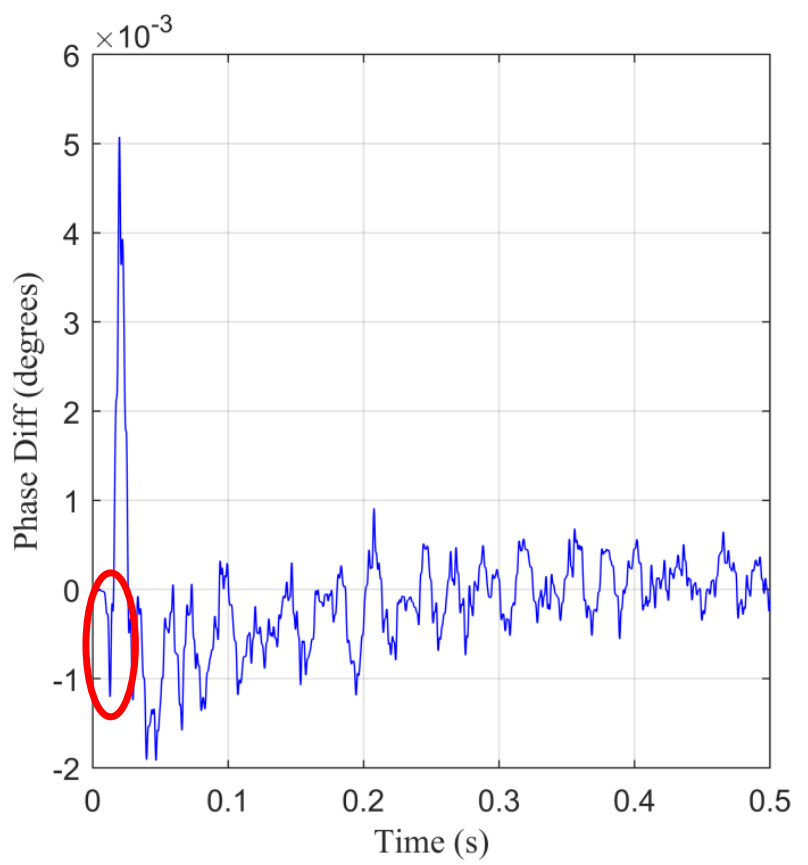

Figure 5: Measured phase difference ( $\propto$ mass flow) for a $1 \mathrm{~ms}$ ET single shot
Figure 6 shows the measured phase difference for a $2 \mathrm{~ms}$ single injection. Note that while the initial peak flows ( $\sim 5 \mathrm{e}-3$ degrees $)$ are similar for both the $1 \mathrm{~ms}$ and $2 \mathrm{~ms}$ ET injections, the subsequent returns towards zero flow are different. In Figure 5 there is a negative offset in the phase measurement which subsides at around $t=0.2 \mathrm{~s}$, approximately $175 \mathrm{~ms}$ after the injection. In Figure 6 there is a positive offset in the phase measurement which subsides at around $\mathrm{t}=0.3 \mathrm{~s}$, approximately $200 \mathrm{~ms}$ after the injection.

As discussed in the next section, where steady pulse trains are applied, the 'zero offset' of the flow measurement stabilizes and can be readily removed.

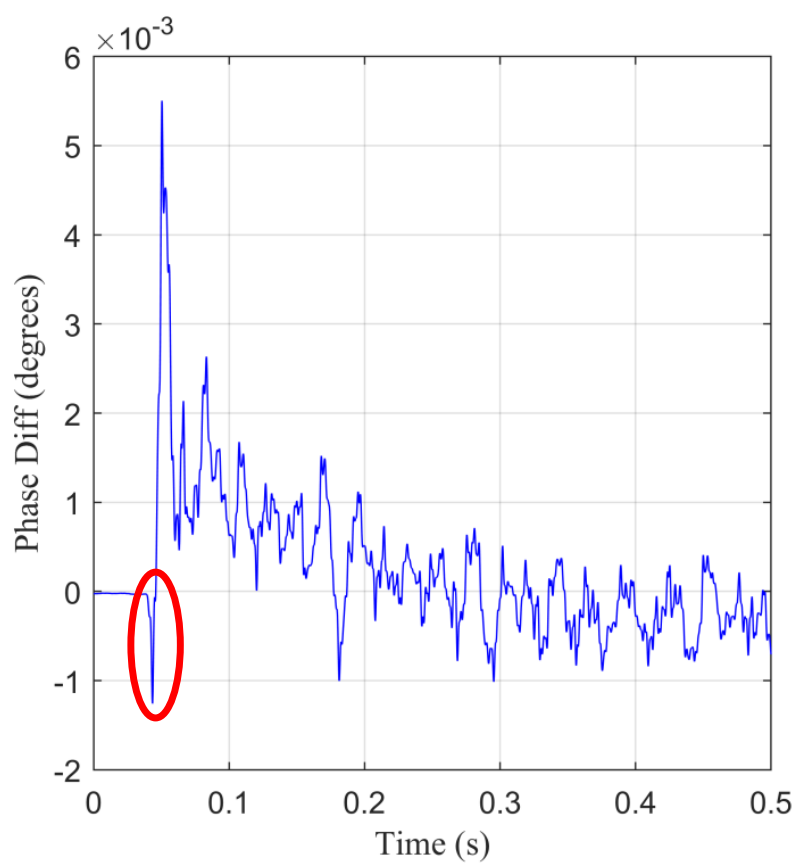

Figure 6: Measured phase difference ( $\propto$ mass flow) for a 2 ms ET single shot

A notable feature of both single shot cases reported here is that the initial mass flow rate is negative (circled areas on Figure 5 and Figure 6) before the clear positive injection mass flow. Given the lack of a return line on GDI injectors, it is likely that what is being observed is initial negative flow associated with the injector needle lifting at start of injection (SOI). It would be expected that the initial flow associated with the injector needle lifting would be away from the injector (i.e. negative). This flow feature is not observed in the previously undertaken diesel tests [9], as those injectors are fitted with a spill, through which such flow would be expected to pass. That this detail can be observed, is a promising result for the future development of the Fast NGC system.

\section{Injection trains}

In order to evaluate the performance of the system under more representative conditions, serial injection trials have been conducted on the test rig at typical injection frequencies for GDI engine operation. As mentioned above, the equivalent engine speeds selected, 1788 and $2978 \mathrm{rpm}$, are one tenth and one sixth

Page 4 of 10 
(respectively) of the forced oscillation frequency of the meter - thus ensuring that each injection occurs at the same flowtube phase to minimize dynamic effects.

Figure 7 and Figure 8 show the measured phase difference for a series of $2 \mathrm{~ms}$ ET injections at 1788 and $2978 \mathrm{rpm}$ respectively. The individual injections are clearly observed by the Fast NGC system; the variation in peak heights observed can be attributed to two factors, firstly a minor variation in the DC of the Fast NGC system, and secondly the previously observed impact of flow tube phase, as the injection frequency is only an approximate multiple of the flow tube drive oscillation frequency. In addition, there are likely to be shot-to-shot variations in injected mass, which the system will also be captured.

Good repeatability is observed across multiple pulses in each injection train. However, the current limitations of the measurement system are demonstrated by a comparison of results for different pulse trains. Note that the peak heights and enclosed areas of these pulse trains at 1788 and $2978 \mathrm{rpm}$ are significantly different to each other. This has the effect of changing the appearance of the behaviour of the phase difference between injections, whereas in fact the behaviour is similar. Both of these observations are attributed to the influence of the instantaneous flowtube phase on the reported phase difference.
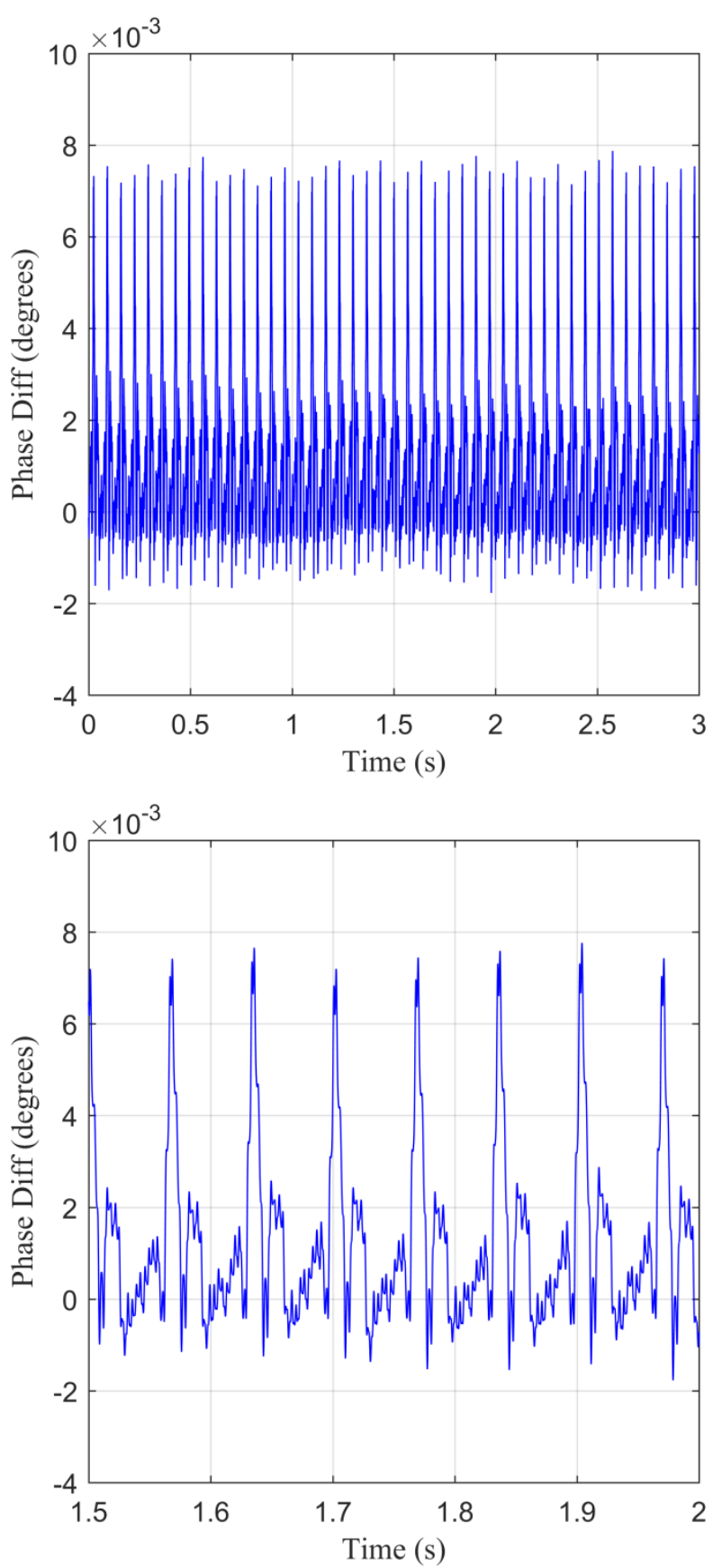

Figure 7: Measured phase difference ( $\propto$ mass flow) for a series of 2 ms ET injections at $1788 \mathrm{rpm}$ equivalent speed - detail of individual injections shown in lower part of figure 

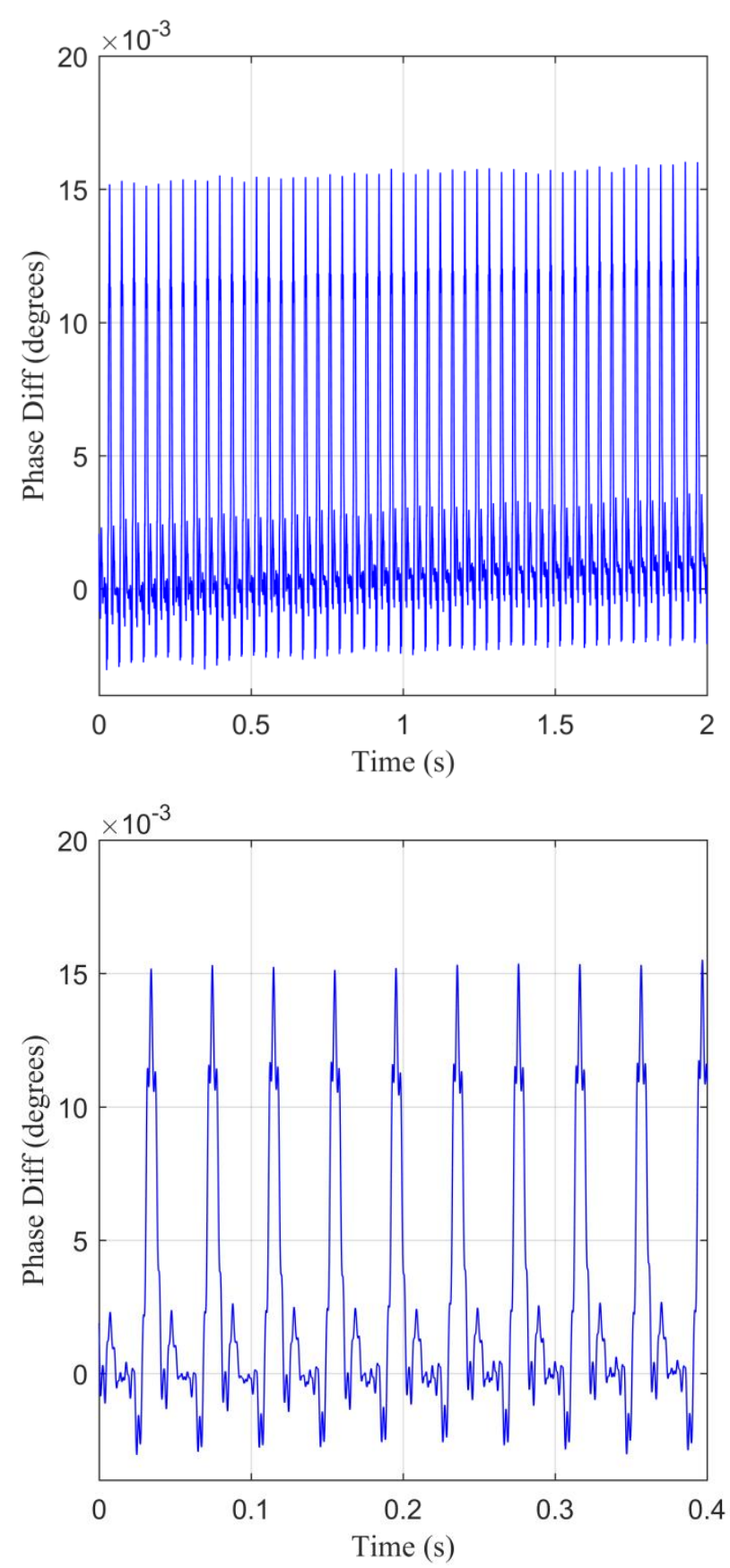

Figure 8: Measured phase difference ( $\propto$ mass flow) for a series of 2 ms ET injections at $2978 \mathrm{rpm}$ equivalent speed - detail of individual injections shown in lower part of figure

\section{Repeatability}

The series of $2 \mathrm{~ms}$ ET injections at $1788 \mathrm{rpm}$ equivalent speed have been analysed for repeatability. As CFM phase difference is, for a fixed fluid density (which is of course temperature dependent) and hence period of oscillation, proportional to the mass flow rate, obtaining the area of an individual injection will give the total injected mass (albeit in arbitrary units of ${ }^{\circ} \mathrm{s}$ ). This is shown in Figure 9. The upper graph of Figure 9 shows area approximations for a subset of the injections; the area is obtained by calculating a Page 6 of 10 "baseline" (a zero level of the noise), finding the peak of each injection and then creating a triangle between those three points. Firstly, the peaks were identified and removed to form a no-peak phase difference signal. The baseline is then calculated using a median filter with a window of $75 \%$ of the injection period on the no-peak phase difference signal. This discretised area is integrated to give a mass value for the injection. The resulting injection areas are shown in the histogram in the lower half of Figure 9; the corresponding distribution is shown in Table 3. The distribution is tight, with a Coefficient of Variation $(\mathrm{CoV})$ of approximately $6.6 \%$.
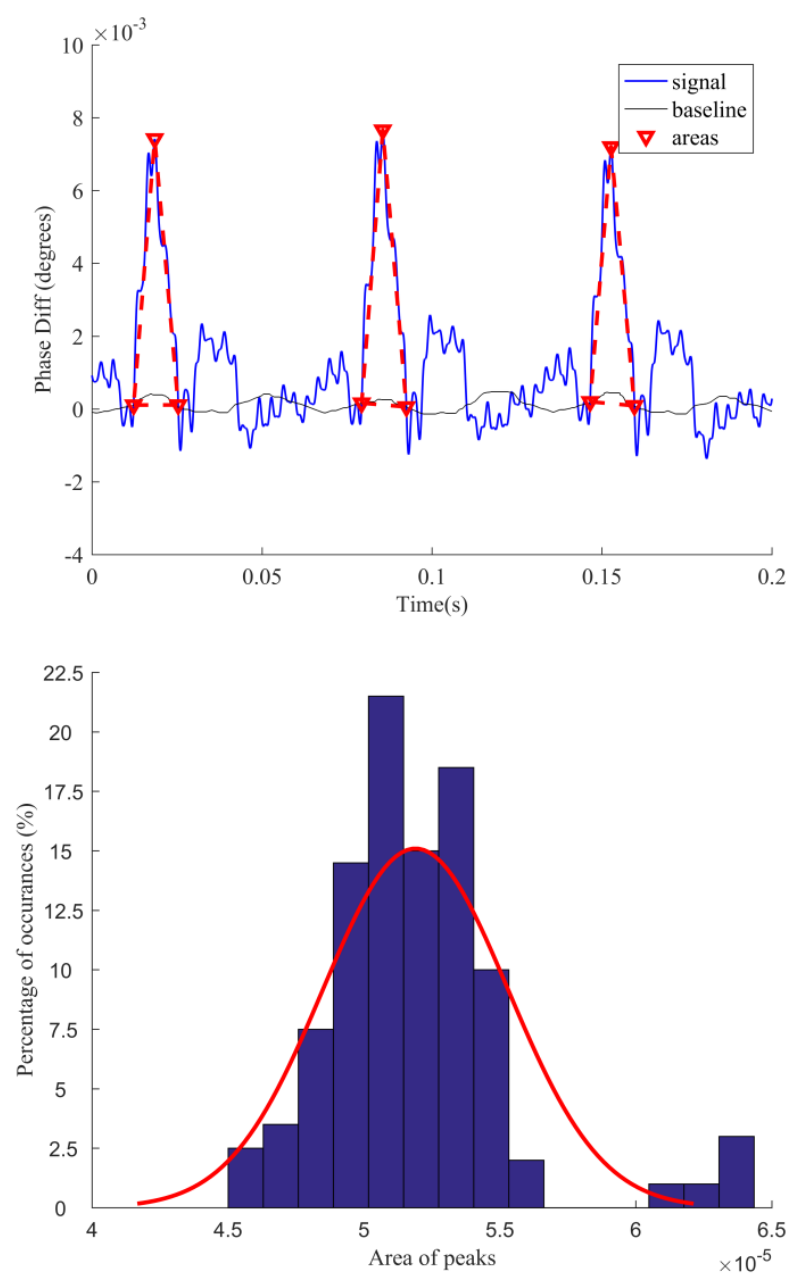

Figure 9: Cumulative mass flow analysis showing calculation area (upper) and histogram of injected masses (lower) for $2 \mathrm{~ms}$ ET injections at $1788 \mathrm{rpm}$

Table 3: Statistical data for a series of $2 \mathrm{~ms}$ ET injections at $1788 \mathrm{rpm}$ equivalent speed

\begin{tabular}{|l|l|}
\hline Number of injections & 200 \\
\hline Mean $\left({ }^{\circ} \mathrm{s}\right)$ & $5.19 \mathrm{e}-05$ \\
\hline Median $\left({ }^{\circ} \mathrm{s}\right)$ & $5.14 \mathrm{e}-05$ \\
\hline Standard deviation $\left({ }^{\circ} \mathrm{s}\right)$ & $3.41 \mathrm{e}-06$ \\
\hline $\mathrm{CoV}(\%)$ & 6.57 \\
\hline
\end{tabular}


Figure 10, Figure 11, and Figure 12 show calculation areas and histograms of integrated areas for 2, 1.5, and $1 \mathrm{~ms}$ (respectively) ET injections at $2978 \mathrm{rpm}$ equivalent speed. The calculations of area have been obtained by the same method as the $1788 \mathrm{rpm}$ case. The corresponding distributions of the histograms are shown in Table 4. Here it can be seen that the measured areas (which are proportional to injected mass) rise with increasing injection duration. Furthermore, as the injected mass increases, the signal to noise ratio (SNR) improves (compare the overall noise levels in Figure 10 and Figure 12) and the $\mathrm{CoV}$ of the injections falls to $0.94 \%$ over 200 injections for the $2 \mathrm{~ms}$ ET injections case. It should be noted that we expect the shot-to-shot $\mathrm{CoV}$ of this injector to be $<1 \%$ which may give some guidance as to the repeatability of our technique at the moment. This demonstrates good repeatability for this test system and is the first time that the repeatability of this technique has been reported.
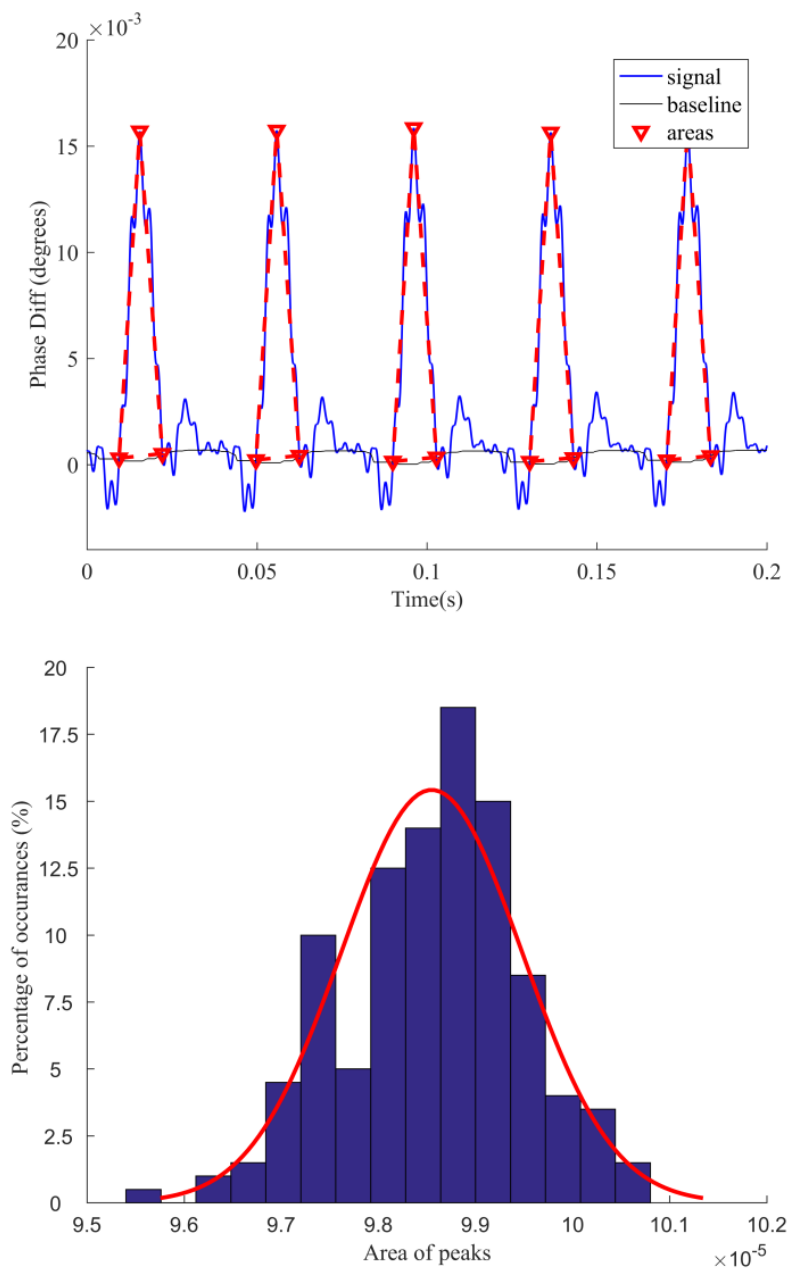

Figure 10: Cumulative mass flow analysis showing calculation area (upper) and histogram of injected masses (lower) for $2 \mathrm{~ms}$ ET injections at $2978 \mathrm{rpm}$
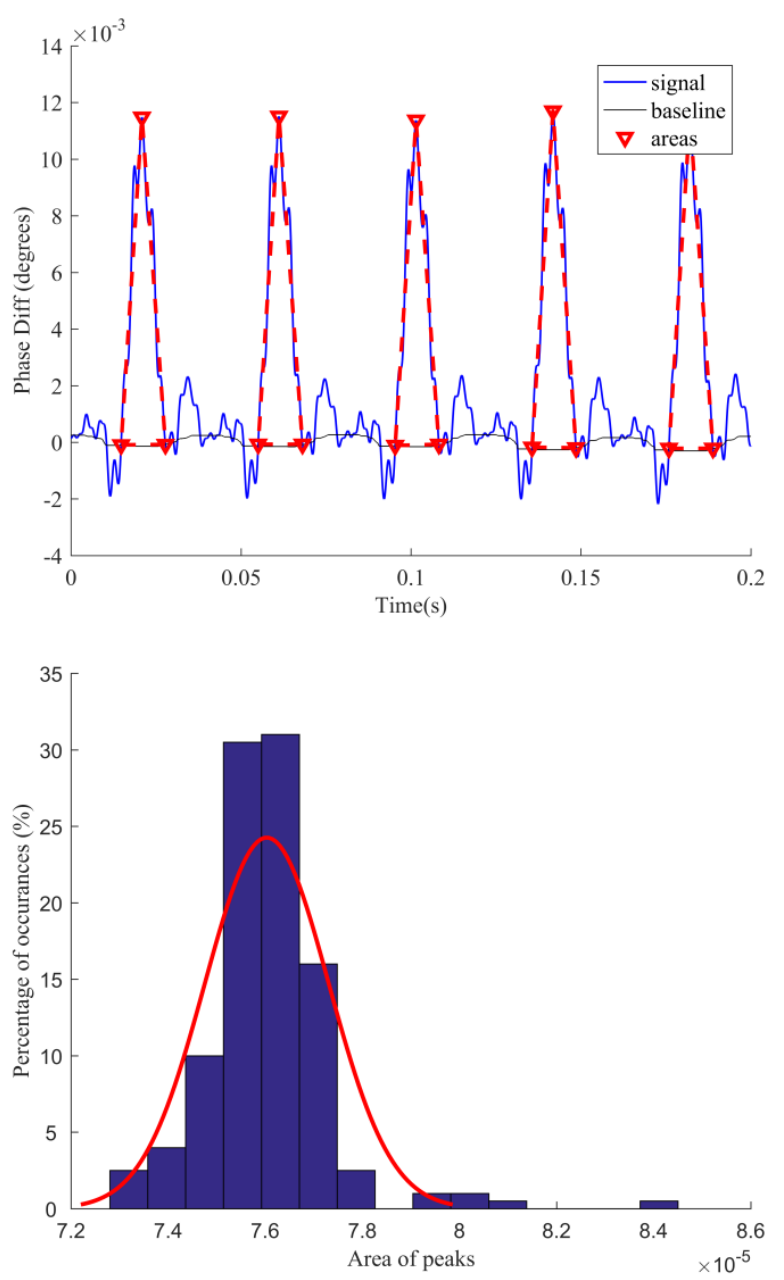

Figure 11: Cumulative mass flow analysis showing calculation area (upper) and histogram of injected masses (lower) for $1.5 \mathrm{~ms}$ ET injections at $2978 \mathrm{rpm}$ 

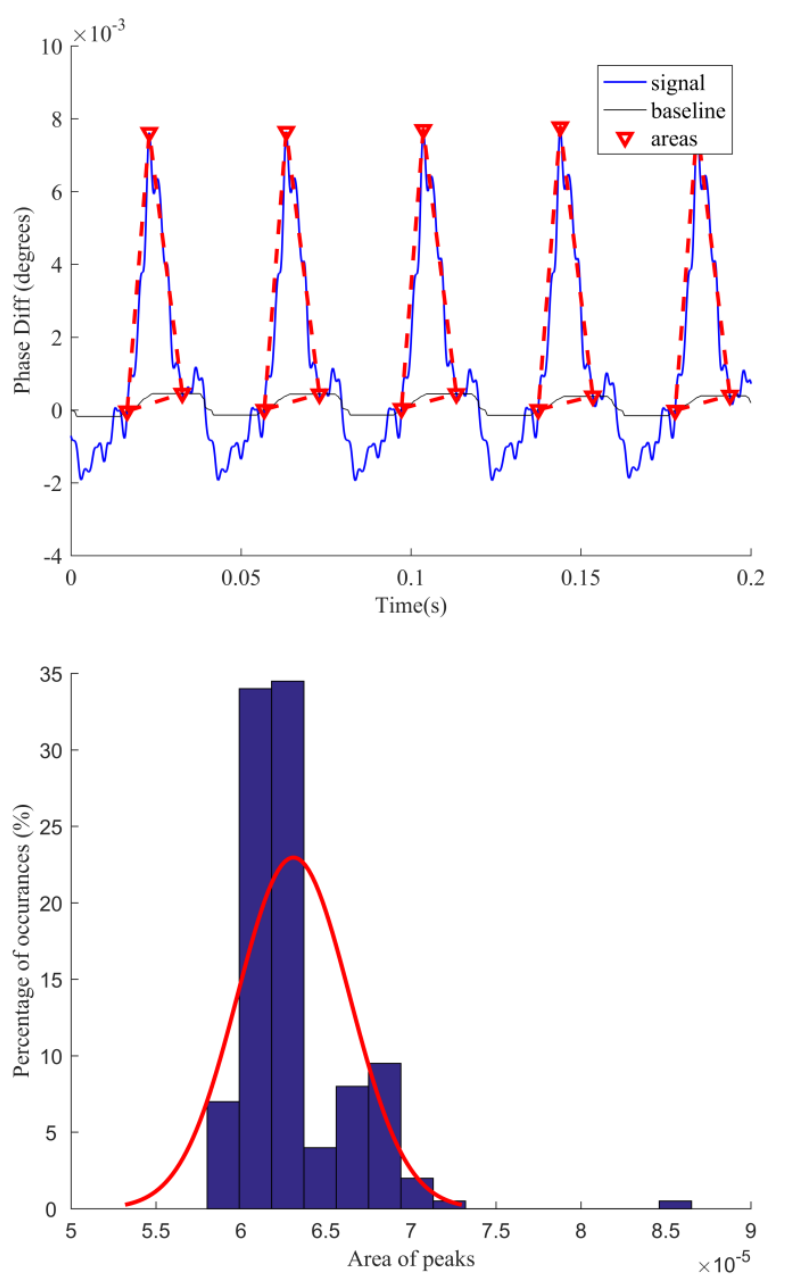

Figure 12: Cumulative mass flow analysis showing calculation area (upper) and histogram of injected masses (lower) for $1 \mathrm{~ms}$ ET injections at $2978 \mathrm{rpm}$

Table 4: Statistical data for series of injections at $2978 \mathrm{rpm}$ equivalent speed

\begin{tabular}{|l|l|l|l|}
\hline Injection ET (ms) & 2 & 1.5 & 1 \\
\hline Number of injections & 200 & 199 & 200 \\
\hline Mean $\left({ }^{\circ} \mathrm{s}\right)$ & $9.86 \mathrm{e}-5$ & $7.60 \mathrm{e}-5$ & $6.31 \mathrm{e}-5$ \\
\hline Median $\left({ }^{\circ} \mathrm{s}\right)$ & $9.87 \mathrm{e}-5$ & $7.63 \mathrm{e}-5$ & $6.22 \mathrm{e}-5$ \\
\hline Standard Deviation $\left({ }^{\circ} \mathrm{s}\right)$ & $9.31 \mathrm{e}-7$ & $1.27 \mathrm{e}-6$ & $3.30 \mathrm{e}-6$ \\
\hline $\mathrm{CoV}(\%)$ & 0.94 & 1.67 & 5.23 \\
\hline
\end{tabular}

\section{Partial injection}

Within a long chain of injections, at an equivalent engine speed of $2978 \mathrm{rpm}$, a partial injection occurred (the injector did not completely inject for a single shot within the injection train). An audible difference was heard between this partial injection and the other injections in the series. This is shown in Figure 13. The Fast NGC system clearly picks up the partial injection, showing there is some initial flow, followed by noise (in the pattern where there is zero

Page 8 of 10 flow) within the signal. This is distinct from the other clearly visible injections within the pulse train. It should be noted that these partial injections are excluded from the $\mathrm{CoV}$ calculations earlier.

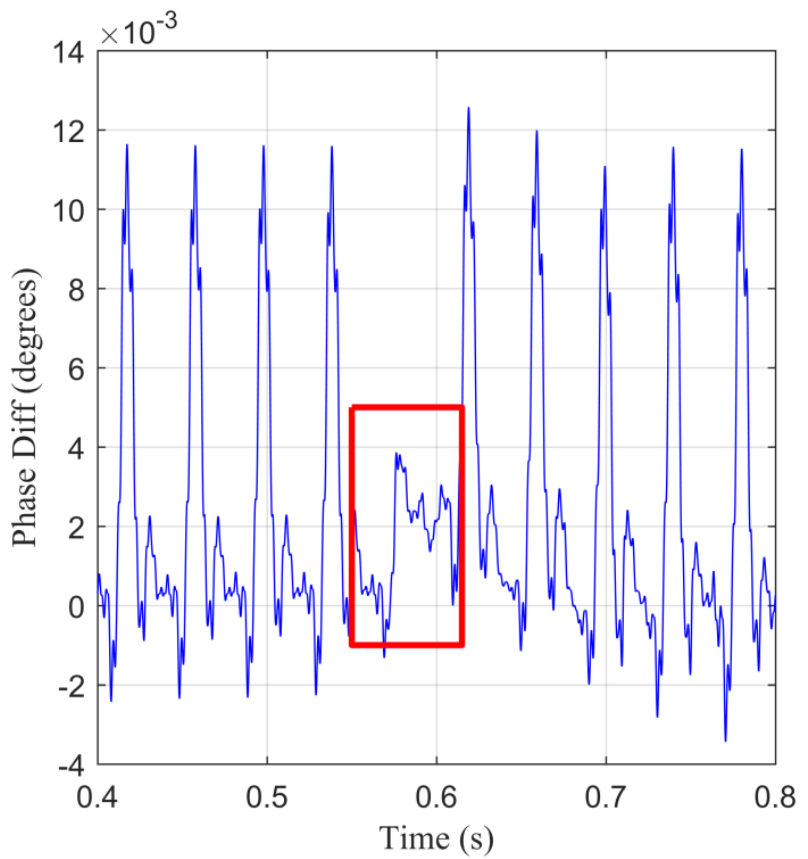

Figure 13: Simulated misfire (red square) within a 2978 rpm equivalent engine speed pulse train of $1.5 \mathrm{~ms}$ ET injections

\section{Influence of flow tube phase}

The influence of flow tube phase, previously observed in [9] has been further investigated in this work. Figure 14 shows a series of $2 \mathrm{~ms}$ ET injections at $14.87 \mathrm{~Hz}$ (which corresponds to an equivalent engine speed of $1784 \mathrm{rpm}$ ) - the injection frequency deliberately chosen to be a slight offset from a multiple of the natural drive mode frequency of the flow tube. It can be seen that after an initial settling period (circled in red), the measured phase difference follows a sinusoidal shape, which is related to the phase of the forced oscillation of the flow tube at the moment that the fuel is injected. During the settling period the amplitude control of the flow tube is adapting to the new flow dynamics, this is followed by the steady state period where the observed phase difference generates a sinusoidal trajectory related to the flow tube phase at the moment of each injection. 


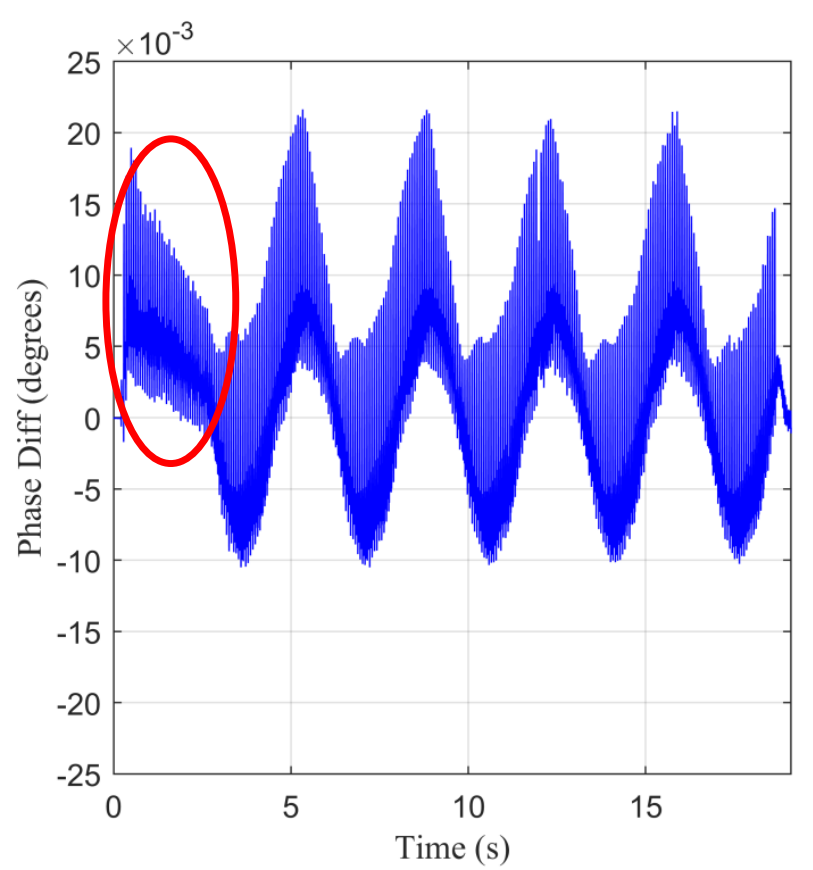
injections at $14.87 \mathrm{~Hz}$ showing impact of flow meter phase
Figure 14: Measured phase difference ( $\propto$ mass flow) for a series of $2 \mathrm{~ms}$ ET

The Prism filters and CFM mounting needed to be modified to account for the significant additional mechanical vibrations caused by the lack of an injector spill (which had been present in previous diesel applications). These mechanical vibrations induced at the end-ofinjection caused significant disturbances to the CFM oscillations and their measurement. A detailed discussion on the modifications necessary to the Prism as a result of this is this will be the topic of a separate publication.

The influence of the instantaneous phase of the flow tube forced oscillation has been further investigated. It has been shown that the oscillation phase directly affects the measured phase difference, and hence observed mass flow rate, but that this DC offset can be discounted for analysis of total injected mass in an injection. Further work will investigate removing the DC offset entirely.

The Fast NGC system has been shown to be viable on GDI injections as well as the previously established viability on diesel. Clearly the results are at an early stage, with accurate measurement of injection rate shape and total injected mass not yet possible. That said, this work has made clear where the future developments need to lie primarily in flow tubes with a higher forced oscillation frequency (of order $\mathrm{kHz}$ ), which will not only enable a wider range of engine operating points to be studied, but also avoid the elongation in time of the measurement observed. Subsequent to that, the next step will be to deploy the Fast NGC system on-engine.

\section{References}

The relatively low forced oscillation frequency of the CFM also means that the observed injection durations from the CFM are elongated in time. All of the injections reported in this work, whether $1 \mathrm{~ms}, 1.5 \mathrm{~ms}$, or $2 \mathrm{~ms}$ ET injections (for example this can clearly be seen in Figure 5 and Figure 6), have a measured duration of approximately $16 \mathrm{~ms}$, arising from a combination of the mechanical response of the flowtube and signal processing delays.. We fully expect that the issue of elongated pulse duration will be resolved by using a flow tube (and matching signal processing) operating at a higher frequency.

\section{Summary / Conclusions}

The Fast NGC system has been deployed on a GDI injector mounted on a rig and tested at a pressure of 150 bar for the first time. The Fast NGC system has been shown to be as capable of identifying GDI injections, as it was in a diesel system as reported in previous work. A number of the features of the Fast NGC system visible in that work were also seen here, as well as some additional features unique to GDI.

Features of GDI injection such as an initial negative mass flow as the injector needle lifts at SOI for a single shot can be clearly observed through the Fast NGC system. The Fast NGC system has also shown its capability for fault detection, by clearly observing a misfire induced in a pulse train of injections.

The repeatability of the Fast NGC system has been assessed for the first time here. It has been found that by discretising injections and summing their areas; CoVs of between $0.94 \%$ and $6.6 \%$ have been observed. Given the preliminary nature of this technique, this is an encouraging starting point. Future developments such as higher frequency flow tubes will assist in reducing this.

Page 9 of 10
1. Zhao, H., Overview of Gasoline Direct Injection Engines, in Advanced direct injection combustion engine technologies and development: Gasoline and gas engines. 2010, Woodhead Publishing Ltd.

2. Eastwood, P., Particulate Emissions from Vehicles. 2008: SAE International and John Wiley \& Sons, Ltd.

3. Leach, F., Knorsch, T., Laidig, C., and Wiese, W., A Review of the Requirements for Injection Systems and the Effects of Fuel Quality on Particulate Emissions from GDI Engines, SAE Technical Paper 2018-01-1710, 2018, https://doi.org/10.4271/2018-01-1710.

4. Bosch, W., The Fuel Rate Indicator: A New Measuring Instrument For Display of the Characteristics of Individual Injection, SAE Technical Paper 660749, 1966, https://doi.org/10.4271/660749.

5. Arcoumanis, C. and Baniasad, M., Analysis of Consecutive Fuel Injection Rate Signals Obtained by the Zeuch and Bosch Methods, SAE Technical Paper 930921, 1993, https://doi.org/10.4271/930921.

6. Payri, R., Gimeno, J., Marti-Aldaravi, P., and Vaquerizo, D., Momentum Flux Measurements on an ECN GDi Injector, SAE Technical Paper 2015-01-1893, 2015, https://doi.org/10.4271/2015-01-1893.

7. $\quad$ Hentschel, L., et al., Der neue 1,0l TSI mit $85 \mathrm{~kW}$ Otto Partikelfilter - saubere, effiziente Performance für den UP! GTI, in 26. Aachener Kolloquium. 2017: Aachen, Germany. p. $971-986$.

8. Baker, R.C., Flow Measurement Handbook: Industrial Designs, Operating Principles, Performance, and Applications. 2 ed. 2016, Cambridge: Cambridge University Press.

9. Leach, F., Davy, M., Henry, M., Tombs, M. et al., A New Method for Measuring Fuel Flow in an Individual Injection in Real Time, SAE Technical Paper 2018-01-0285, 2018, https://doi.org/10.4271/2018-01-0285. 
10. Leach, F., et al., Fast Coriolis Mass Flow Metering for Monitoring Diesel Fuel Injection. Flow Measurement and Instrumentation, 2017. 58: p. 1-5.

11. Henry, M., O. Bushuev, and O. Ibryaeva, Prism Signal Processing for Sensor Condition Monitoring, in IEEE International Symposium on Industrial Electronics. 2017: Edinburgh, UK.

12. Henry, M., et al., The Prism: Efficient Signal Processing for the Internet of Things. IEEE Industrial Electronics Magazine, 2017. 11(4): p. 22-32.

13. Henry, M.P., Method and system for tracking sinusoidal wave parameters from a received signal that includes noise. GB Patent Application GB1619086.0, 11 November 2016.

14. Henry, M.P., An Introduction to Prism Signal Processing Applied to Sensor Validation. Measurement Techniques, 2018. 60(12): p. 1233-1237.

15. Mohd Murad, S., Camm, J., Davy, M., Stone, R. et al., Spray Behaviour and Particulate Matter Emissions with M15 Methanol/Gasoline Blends in a GDI Engine, SAE Technical Paper 2016-01-0991, 2016, https://doi.org/10.4271/2016-01-0991.

16. Sandford, M., Page, G., and Crawford, P., The All New AJV8, SAE Technical Paper 2009-01-1060, 2009, https://doi.org/10.4271/2009-01-1060.

\section{Contact Information}

Felix Leach,

Dept of Engineering Science

University of Oxford

Parks Rd

Oxford

OX1 3PJ

UK

felix.leach@eng.ox.ac.uk

\section{Acknowledgments}

The authors would like to thank BP International for funding this work. The authors would also like to acknowledge Rheonik GmbH for support.

\section{Definitions/Abbreviations}

CFD

CFM

CoV

EOI

ET

FIR

GDI

NGC

PM

R\&D

SNR

SOI

VW
Computational Fluid Dynamics

Coriolis Flow Meter

Coefficient of Variation

End of injection

Energising time

Finite Impulse Response

Gasoline Direct Injection

Next Generation Coriolis

Particulate Matter

Research and Development

Signal to Noise Ratio

Start of injection

Volkswagen 